\title{
2285. Non-uniform embedding applications for synchronization analysis
}

\author{
Migle Drulyte $^{1}$, Kristina Lukoseviciute ${ }^{2}$, Jurate Ragulskiene ${ }^{3}$ \\ Department of Mathematical Modeling, Kaunas University of Technology, Kaunas, Lithuania \\ ${ }^{1}$ Corresponding author

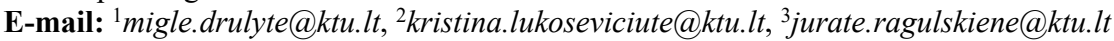 \\ Received 25 October 2016; received in revised form 4 December 2016; accepted 9 December 2016 \\ DOI https://doi.org/10.21595/jve.2016.18090
}

\begin{abstract}
Iterative polynomial model for synchronization of vibrations was proposed. A polynomial was constructed using time delay coordinates. For time delay kernel estimation, a non-uniform embedding was used. Evolutionary optimization algorithms were introduced for non-uniform time delay identification. Performance investigation of proposed method was done for two different vibrations.
\end{abstract}

Keywords: synchronization of vibration, non-uniform embedding, evolutionary optimization algorithms.

\section{Introduction}

The synchronization phenomena have been intensively investigated due to its potential application in physical processes like communications [1, 2], system control [3], artificial analysis $[4,5]$, financial time series [6]; and biological processes, like neurophysiological systems [7] and cardiorespiratory rhythms [8]. Usually synchronization theory is based on phase synchronization $[5,9]$. However, state space of the system can be reconstructed from their observations [10] and various geometrical and dynamic properties can be analyzed [11] and successfully applied in synchronization analysis [3, 12]. In past, a variety of the synchronization methodologies of chaotic vibration systems have been suggested, such as variable structure control approach, back stepping control approach and others $[13,14]$.

One of the main problems arising when designing of dynamical model is instability of a system $[13,15]$. Assessing model based on its dynamic response requires synchronous response measurements. For modeling of dynamic synchronization the rules that governs the original system is required. To find those rules non-uniform embedding and polynomial approximation model have been invoked. Systems embedding is an important step in the process of modeling of a system [16]. Attractor embedding is used to characterize the dynamics of a true system represented by obtained vibration. The embedding theorem says that dynamical systems has the same topological properties and is better represented by delayed version of an observed vibration [17]. Embedding dimension and time delay are two parameters determining the reconstruction of a system by a delay coordinate method. There are several ways to determine embedding dimension like correlation dimension or neural networks $[18,19]$. In this paper one of the classical methods called false nearest neighbor (FNN) method for identification of embedding dimension is used [20]. For identification of time delays computational techniques like mutual information or autocorrelation based methods often used [21]. However, in this paper we propose a simple way for time delay selection. For time delay identification, evolutionary optimization algorithms were introduced [16]. The problem of evolutional optimization is to identify optimal set of time delay that represents the best properties of reconstructed system. Polynomials approximation was used to construct the synchronization of vibrations [22]. Polynomials produce a good approximation to complex systems [23]. Polynomial model is derived using optimal non-uniform time delay coordinates.

For synchronization task we propose new iterative algorithm for polynomial construction. The synchronization was examined for vibration obtained by aquarium with air pump [24] and wind turbine pinion gear [25]. The model of synchronization is constructed in several steps. In Section 2 
the signal reconstruction based on non-uniform embedding proposed in [16] was applied. Evolutionary optimization algorithms for identification of the optimal set of time delays are discussed in Section 3. In Section 4 synchronization model was defined. Simulation results for two vibrations are presented in Section 5. And concluding remarks are discussed in the final section.

\section{Non-uniform embedding reconstruction of a signal}

Let us consider discrete time series which is presented in form of $x_{1}, x_{2}, \ldots, x_{N}$. The non-uniform reconstruction is obtained from original time series when the time delays between coordinates were chosen non-uniformly $Y_{i}=\left\{x_{i}, x_{i+\tau_{1}}, x_{i+\tau_{1}+\tau_{2}}, \ldots, x_{i+\sum_{k=1}^{d-1} \tau_{k}}\right\}$, $i=1,2, \ldots, N-\sum_{k=1}^{d-1} \tau_{k}$. The embedding dimension is denoted by $d ; Y_{i}$ is the reconstructed vector with dimension $d ; \tau_{k} \in N$ are the time delays.

First task of the reconstruction is to determine optimal embedding dimension. For this purpose the false nearest neighbors method was applied [20]. In this method, if two points become distant by increasing embedding dimension $d$ then one of them is supposed as false. Embedding dimension is chosen when there is no false nearest neighbours. The nearest neighbor in the $(d-1)$-th cycle is supposed as false if:

$\frac{R_{d+1}^{2}(i)-R_{d}^{2}(i)}{R_{d}^{2}(i)}>R_{t o l}$

where $R_{d}^{2}(i)$ is the Euclidean distance of the $i$-th point in the $(d-1)$-th cycle to its nearest neighbour, $R_{d+1}^{2}(i)$ is the same distance in the $d$-th cycle with additional $x\left(t-\tau_{d}\right)$ time delay coordinate and $R_{\text {tol }}$ is a threshold [20].

The next step of time series embedding is to choose algorithm which helps to find the optimal set of time delays. This set gives best characteristics of the reconstructed attractor in $d$-dimensional space. For this case we use the objective function which was used for non-uniform attractor embedding in [16]:

$F\left(\tau_{1}, \tau_{2}, \ldots, \tau_{d-1}\right)=\frac{\pi}{2} \frac{\int_{0}^{\infty} A(\omega) Q\left(\tau_{1}, \tau_{2}, \ldots, \tau_{d-1}, \omega\right) d \omega}{\int_{0}^{\infty} A(\omega) d \omega}$

where $Q\left(\tau_{1}, \tau_{2}, \ldots, \tau_{d-1}, \omega\right)$ is the embedding quality function Eq. (3) of reconstruction into time delay phase space. This quality function evaluates arithmetic average of all planar projection of reconstructed attractor [16]. Thus, if time series is reconstructed into $d$-dimensional time delay space there are $d(d-1) / 2$ planar projection and quality function can be expressed as:

$$
\begin{aligned}
& Q\left(\tau_{1}, \tau_{2}, \ldots, \tau_{d-1}, \omega\right) \\
& \quad=\frac{2}{d(d-1)}\left(\sum_{i=1}^{d-1}\left|\sin \left(\omega \delta \tau_{i}\right)\right|+\sum_{i=1}^{d-2}\left|\sin \left(\omega \delta\left(\tau_{i}+\tau_{i+1}\right)\right)\right|+\cdots+\left|\sin \left(\omega \delta \sum_{j=1}^{d-1} \tau_{j}\right)\right|\right) .
\end{aligned}
$$

The maximum of the objective function Eq. (2) gives the optimal set of time delays $\left\{\tau_{1}, \tau_{2}, \ldots, \tau_{d-1}\right\}$. With this set of time delays chaotic attractor in the reconstructed space have optimal geometric properties. 


\section{Evolutionary algorithms for the identification of an optimal set of non-uniform time delays}

As it was mentioned in previous section the non-uniform embedding can express the best topological properties of reconstructed signal [17]. Although this reconstruction is affective, but in this case an optimized set of all time delays $\tau_{i}(i=1,2, \ldots, d-1)$ must be identified. If the upper bound $T$ for possible numerical values of time delays are chosen, then the number of different combinations of time delays is the number of different combinations without permutations is $(T+d-2) ! /((d-1) !(T-1) !)$ [16]. For example, there is 292825 different combinations of time delays without permutations when $d=5$ and $T=50$. For higher dimension, it is infeasible to use full sorting. To solve this problem evolutionary optimization algorithms where used for identification for time delays. In this paper we use three different optimization algorithms which were adapted for identification of optimal set of non-uniform time delays.

\subsection{Genetic algorithms for the identification of an optimal set of non-uniform time delays}

The first evolutionary algorithm used for optimization is Genetic Algorithm (GA) proposed by John Holland in 1975 [26]. It is an adaptive heuristic search algorithm that is widely used in various optimization problems. Genetic algorithm consists of four main steps: initialization, selection, crossover operation and mutation [27].

1) Initialization. For identification of non-uniform time delays the GA algorithm is constructed in a way that every chromosome represents an array of time delays (genes). Every chromosome $c r_{i}=\left\{\tau_{1}^{(i)}, \tau_{2}^{(i)}, \ldots, \tau_{d-1}^{(i)}\right\}$ is of length $(d-1)$ and every gene is an integer number [17]. The initial population of $\mathrm{n}$ chromosomes $\left\{c r_{1}, c r_{2}, \ldots, c r_{n}\right\}$ is generated randomly. Then the objective function describe by Eq. (2) is maximized.

2) Selection. For selecting of chromosomes random roulette method was used [27]. The higher is the objective function value the higher is a chance for chromosome to be selected for the next generation.

3) Crossover. All selected chromosomes are grouped into pairs. The crossover between two chromosomes is executed. The modified one-point algorithm is used in crossover operation [17].

4) Mutation. After crossover mutation operation is applied. In other words random number for every gene is generated in the $k$-th generation and if the random number is lower than $\mu$ (usually the value of mutation parameter $\mu<0.01$ ) then that gene is changed by a random integer.

These steps are repeated until maximum number of generations is achieved.

\subsection{Artificial bee colony algorithms for the identification of an optimal set of non-uniform time delays}

The second optimization algorithm used for time delay identification is Artificial Bee Colony $(\mathrm{ABC})$ algorithm $[28,29]$. ABC is a metaheuristic algorithm introduced by Karaboga in 2005. This optimization algorithm is based of improvement of solution in every step of ABC algorithm. $\mathrm{ABC}$ has three parameters: the number of a food source (population), predetermined number of cycles (limit) and stopping criteria (maximum number of generation) [29]. Artificial bee colony optimization algorithm consists of four main phase: initialization, employed bee phase, onlooker bee phase and scout bee phase.

1) Initialization. For identification of non-uniform time delays the $A B C$ algorithm is constructed in a way that every food source represents an array of time delays (food source position). Every food source $s_{i}=\left\{\tau_{1}^{(i)}, \tau_{2}^{(i)}, \ldots, \tau_{d-1}^{(i)}\right\}$ is of length $(d-1)$ and every position $\tau_{k}^{(i)}$, $k=1,2, \ldots, d-1$ is an integer number. The initial population of $n$ food sources $\left\{s_{1}, s_{2}, \ldots, s_{n}\right\}$ is generated randomly.

2) Employed bee phase. The position of the food source is updated by Eq. (4) and the profit 
value for each food source is evaluated:

$V_{i j}=s_{i j}+\phi_{i j}\left(s_{i j}-s_{k j}\right)$

where $\phi_{i j}\left(s_{i j}-s_{k j}\right)$ is a step size, $\phi_{i j}$ is a random number between $[-1 ; 1], k \in(1,2, \ldots, n)$ and $j \in(1,2, \ldots, d-1), k \neq j$ are two randomly chosen numbers.

3) Onlooker bee phase. The selection of food source is performed to obtain best sets of time lags for optimization of the objective function. The food source is selected with probability value $p_{i}$ Eq. (5) associated with that food source and is updated by Eq. (4):

$p_{i}=\frac{F\left(s_{i}\right)}{\sum_{n} F\left(s_{n}\right)}$

where $n$ is the number of the food sources and $F\left(s_{i}\right)$ is the value of objective function of the $i$-th solution in population. The new better solution replaces the old one.

4) Scout bee phase. In the last scout bee phase, employed bees whose solutions cannot be improved after a predefined number of trials become scouts and their solutions are discarded [28]. Scout bees randomly search for a new food source by Eq. (4). Scout bee phase is used only if the position of the food source cannot be improved.

These steps are repeated until maximum number of cycles is reached. The result is set of time lags that give maximum value of the objective function.

\subsection{Cuckoo search algorithms for the identification of an optimal set of non-uniform time delays}

Cuckoo search (CS) is metaheuristic search algorithm introduced by Xin-she Yang and Suash Deb in 2009 [30]. The initial number of cuckoos can lay one egg at the time in randomly chosen nest. The best nests with high objective function values are carried to the next generation. Eggs which are more similar to host nest eggs have a bigger chance to survive others are detected by host bird and thrown away. The grown eggs show the surviving rate in those nests [31]. Cuckoo search algorithm consists of three main steps:

1) Initialization. The CS algorithm is constructed in a way that every nest represents an array of time delays (nest position). Every nest $c_{i}=\left\{\tau_{1}^{(i)}, \tau_{2}^{(i)}, \ldots, \tau_{d-1}^{(i)}\right\}$ is of length $(d-1)$. The initial population of $n$ nests $\left\{c_{1}, c_{2}, \ldots, c_{n}\right\}$ is generated randomly.

2) Phase 1. The objective value of randomly chosen nest is compared with the objective value of the new generated solution by Levy flights Eq. (6). If the new solution is better it replaces the chosen solution:

$V_{i j}=c_{i j}+\alpha \oplus \operatorname{Lévy}(\lambda)$,

where $c_{i j}$ is randomly chosen $j$-th time lag from $c_{i}$ nest, $\alpha>0$ is the step size scaling factor. In most cases $\alpha=1$. The product $\bigoplus$ means entrywise multiplication. In Cuckoo Search algorithm random walks are provided by Levy flights. In Levy flights the step length is estimated using Levy distribution:

Lévy u $=t^{-\lambda}, \quad(1<\lambda<3)$.

3) Phase 2. The part of worst nests with probability $p_{a} \in[0 ; 1]$ are abandoned and new solutions are generated using Eq. (6). The solutions are ranked and the best one solution is found.

These steps are repeated until maximum number of generation is reached. 


\section{Polynomial synchronization}

Time series reconstruction into time delay space allows obtaining certain properties of attractor that can be used in system control. In most of these tasks synchronization of the system is needed. In this paper the synchronization problem is considered as a construction of a model for some dynamical system. Lets $g$ denote the dynamics of the time series:

$x_{i+1}=g\left(X_{1 i}, X_{2 i}, \ldots, X_{d i}\right), \quad i=N+1, N+2, \ldots, K$,

where $X_{1 i}=x_{i}, X_{2 i}=x_{i+\tau_{1}}, \ldots, X_{d i}=x_{i+\sum_{k=1}^{d-1} \tau_{k}}$ is the time delayed vectors with dimension $d$ and set of time delays $\left\{\tau_{1}, \tau_{2}, \ldots, \tau_{d-1}\right\}$. However, the rule $g(\cdot)$ is unknown. In construction of synchronization model the principal rule is to find approximate function $\mathrm{g}$ which would be as close as possible to original dynamics $x$ (note that $x_{N+1}, x_{N+2}, \ldots$ are known). Aim is to minimize the difference:

$E=\min \sum_{i=N}^{K-1}\left\|x_{i+1}-g\left(X_{1 i}, X_{2 i}, \ldots, X_{d i}\right)\right\|$.

For construction of rule $g$ polynomials that provide good approximation to $x$ with proper structure selection were used $[12,22]$. The number of terms in the polynomial expansion of $g$ grows with degree $n$ of the polynomial. There is $M=\sum_{i=1}^{n} \frac{(i+d-1) !}{(i) !(d-1) !}+1$ terms for polynomial of degree $n$ with $d$ variables. For example, there is 10 terms for polynomial of degree $n=2$ and reconstruction dimension of $d=3$.

We consider the $n$-th order polynomial:

$P_{n}\left(X_{1 i}, X_{2 i}, \ldots, X_{d i}\right)=\omega_{0}+\omega_{1} X_{1 i}+\omega_{2} X_{2 i}+\cdots+\omega_{d} X_{d i}+\omega_{d+1} X_{1 i} X_{2 i}+\cdots+\omega_{M} X_{d i}^{n}$,

where $\omega_{0}, \omega_{1}, \ldots, \omega_{M}$ is the coefficients of the polynomial and $X_{k i}(k=1,2, \ldots, d)$ time delay coordinates. The task is to find such $\omega_{0}, \omega_{1}, \ldots, \omega_{M}$ values that the overall solution minimizes error $E$ described by Eq. (9). Polynomial regression model using linear least square techniques based on orthogonal triangular factorization with column pivoting was applied [32]. However, with high embedding dimension and high polynomial order the terms of polynomial also increases. In order to decrease the number of polynomial terms the new iterative reconstruction algorithm was applied.

The iterative polynomial approximation algorithm consist of two steps:

1-step. The second order polynomial $P_{2}\left(X_{1 i}, X_{2 i}, \ldots, X_{d i}\right)$ is constructed and coefficient $\omega_{0}$, $\omega_{1}, \ldots, \omega_{M}$ are optimized.

2-step. Increase the order of the predefined polynomial, by using Eq. (10) with additional variable, which is the second order polynomial constructed in first step:

$P_{4}\left(X_{1 i}, X_{2 i}, \ldots, X_{d i}\right)=P_{2}\left(X_{1 i}, X_{2 i}, \ldots, X_{d i}, P_{2}\left(X_{1 i}, X_{2 i}, \ldots, X_{d i}\right)\right)$.

This step is repeated until the error $E$ does not improve. Finally, the synchronization model is the last polynomial that is found after the optimization process:

$x_{i+1}=P_{2 n}\left(X_{1 i}, X_{2 i}, \ldots, X_{d i}\right)$.

For even order polynomial construction first order polynomials should be used in first step. Thus, in the same synchronization problem we optimize two polynomials of odd and even orders one-at-a-time. The final solution is the lower order polynomial which gives the minimal errors. 


\section{Experiments}

The proposed method for signal synchronization based on non-uniform embedding works well with different vibrations. We will test this technique for a few different vibrations and then make some generalizations.

First for both vibrations Savitzky-Golay Filter was used [33]. The key idea of Savitzky-Golay Filter is that the smoothed value $z_{i}$ in the point $x_{i}(i=1,2, \ldots, N)$ is obtained by taking an average of the neighboring data. More generally we can also fit a polynomial through a fixed number of points. Then the value of the polynomial at $x_{i}$ gives the smoothed value $z_{i}$.

Case 1. First vibration used for this technique is vibration of aquarium with air pump [24]. Length of a vibration is $51 \mathrm{~s}$ and sampling rate is $100 \mathrm{~Hz}$. Every vibration can be efficiently transformed in electrical energy and employed to power electronic devices. In order to achieve this and make a better use of energy the dynamics of vibration must be known.

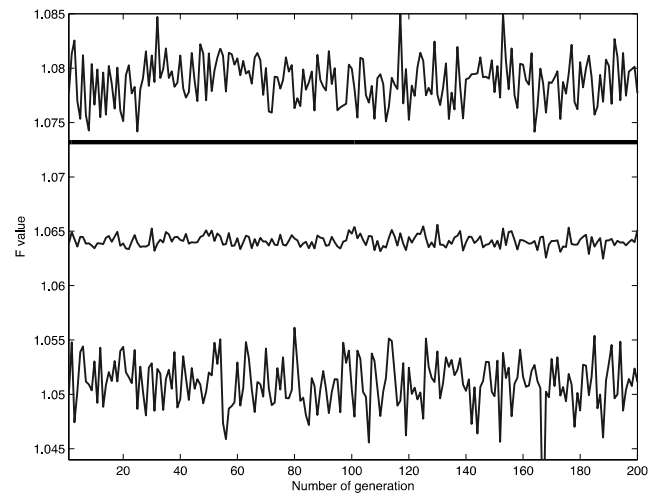

a) CS algorithm

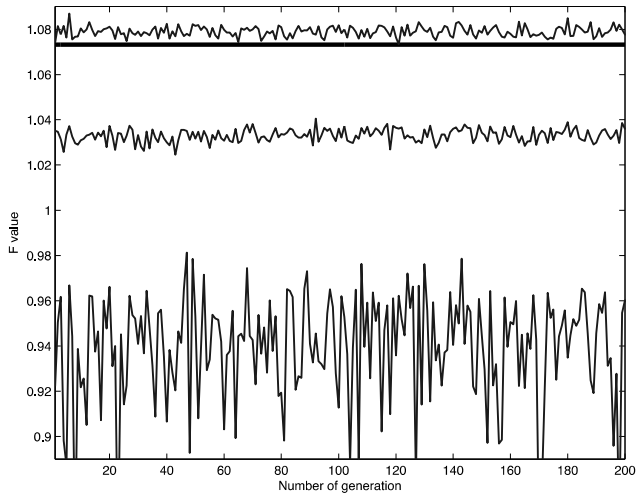

b) ABC algorithm

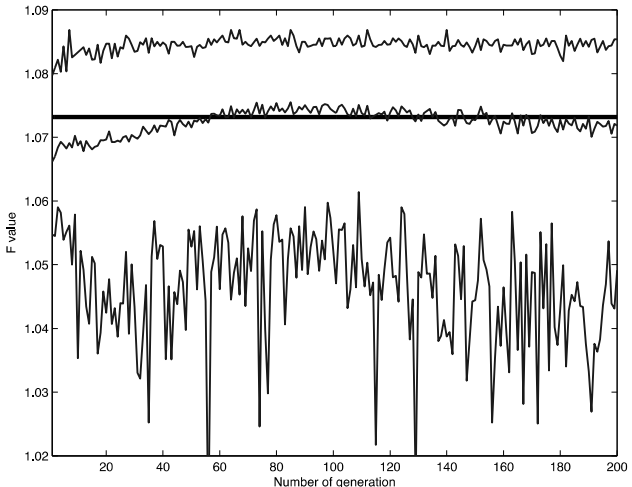

c) GA algorithm

Fig. 1. Objective function value compared to number of generation. The thick line shows objective function value for uniform embedding. Upper thin line - is the maximum value of $F$ for each generation. Middle thin line - is the mean value of $\mathrm{F}$ for each generation.

Lower thin line - is the minimum value of $F$ for each generation

First task of proposed model is to prepare the vibration for synchronization. For noise reduction Savitzky-Golay Filter was applied [33]. The second step was reconstruction into nonuniform time delay phase space. The False Nearest Neighbors algorithm suggested that embedding dimension is $d=6$. With next step the set of optimal time delays was identificated by evolutionary optimization algorithm. Refer to Fig. 1 for objective function value comparison to a number of generation for each optimization algorithm. The number of initial population for each algorithm was chosen equally. In each generation, all three-optimization algorithm were executed 
for 100 times. The thick line in Fig. 1 shows objective function value of uniform embedding. The thin lines show non-uniform embedding objective function value for each generation. As Fig. 1, shows each algorithm managed to find better objective function value than using uniform embedding.

The optimal set of time delays for uniform embedding is determined to be $F(9,9,9,9,9)=1.0732$. The non-uniform set of time delay is determined using evolutionary algorithms shown in Table 1. The set of time delay was chosen with the highest objection function value. This was obtained by Cuckoo Search algorithm. The optimal set for non-uniform embedding is determined to be $F(9,6,6,6,10)=1.0851$.

Refer to Fig. 2 for signal reconstruction into 3-dimensional time delay space using $X_{1}, X_{2}, X_{3}$.

Table 1. Evolutionary algorithm comparison for non-uniform embedding

\begin{tabular}{|c|c|c|c|}
\hline Cases & Evolutionary algorithm & Set of time delays $\left(\tau_{1}, \ldots, \tau_{d-1}\right)$ & $F\left(\tau_{1}, \ldots, \tau_{d-1}\right)$ \\
\hline \multirow{3}{*}{ Case 1} & CS & $(9,6,6,6,10)$ & $F(9,6,6,6,10)=1.0851$ \\
\cline { 2 - 4 } & ABC & $(11,11,8,11,12)$ & $F(11,11,8,11,12)=1.0849$ \\
\cline { 2 - 4 } & GA & $(9,6,6,6,9)$ & $F(9,6,6,6,9)=1.0846$ \\
\hline \multirow{3}{*}{ Case 2 } & CS & $(14,13,15)$ & $F(14,13,15)=1.1349$ \\
\cline { 2 - 4 } & ABC & $(14,14,13)$ & $F(14,14,13)=1.1059$ \\
\cline { 2 - 4 } & GA & $(14,13,17)$ & $F(14,13,17)=1.1027$ \\
\hline
\end{tabular}

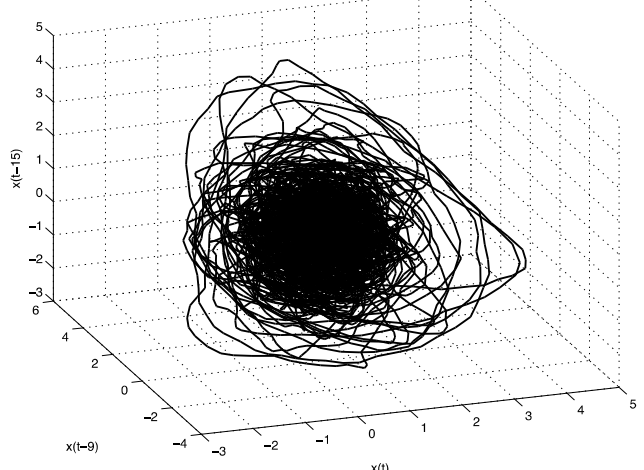

Fig. 2. Vibration reconstructed into 3-dimensional space with time delay set $\left(X_{1}, X_{2}, X_{3}\right)$ and using time delays $\tau_{1}=9$ and $\tau_{2}=6$

The 4-th order polynomial was build with found delays for this vibration. The found model have been given in Appendix A.1. The synchronization for real data and constructed polynomials is shown in Fig. 3. For polynomial approximation, we use 36 terms (while traditional model with polynomial order of 4 and reconstruction dimension $d=6$ would have 210 terms).

Case 2. Second vibration is radial vibration which measurements were taken on $3 \mathrm{MW}$ wind turbine pinion gear. [25] Recorded length of a vibration is 6s and sampling rate is $97656 \mathrm{~Hz}$. In this case we have high vibration levels. The same process was applied to this vibration as in Case 1. First Savitzky-Golay Filter was used to reduce noise of a signal. The False Nearest Neighbors algorithm suggested that embedding dimension is $d=4$. The non-uniform set of time delay is determined using evolutionary algorithms shown in Table 1 . The best set of non-uniform embedding was found with Cuckoo Search algorithm $F(14,13,15)=1.1349$. Refer to Fig. 4 for synchronization results. The 6-th order polynomial was build with found delays for this vibration. The found model have been given in Appendix A.2. The found model has a total 27 terms (while traditional model with polynomial order of 6 and reconstruction dimension $d=4$ would have 210 terms).

As expected synchronization performance was not high due to the complex nature of the vibrations (refer Fig. 4 for residual). Filtering reduces the complexity of vibration and improves 
synchronization results.

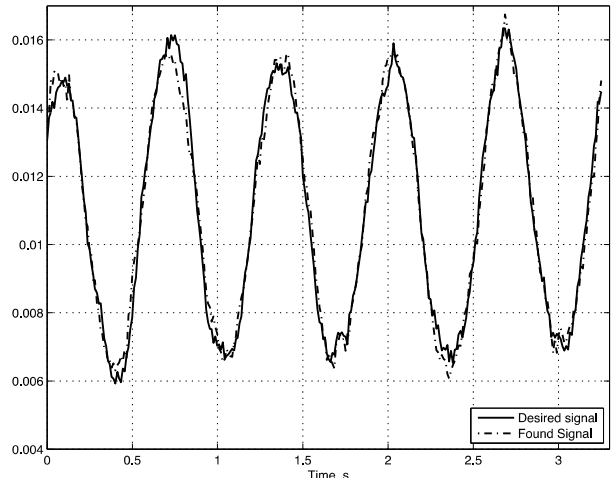

a)

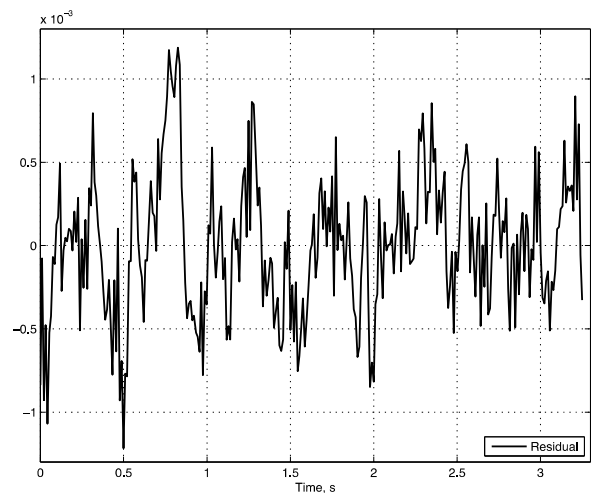

b)

Fig. 3. a) Synchronization of real vibration (solid line) and vibration obtained with polynomial model (dashed line), b) residual of obtained and real signal

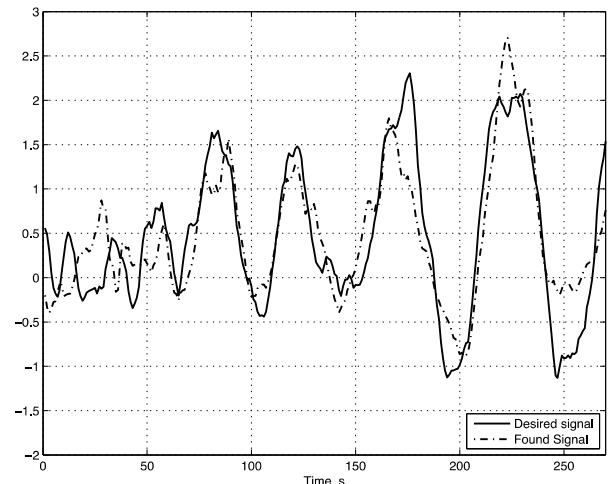

a)

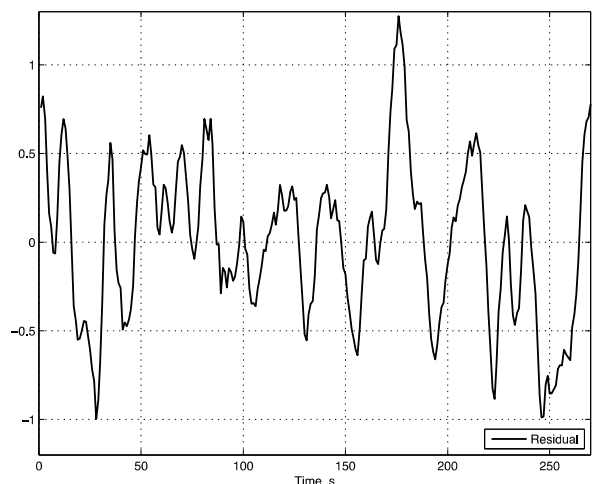

b)

Fig. 4. a) Synchronization of real vibration (solid line) and vibration obtained with polynomial model (dashed line), b) residual of obtained and real signal

\section{Conclusions}

It was demonstrated that synchronization of true dynamics and learnt model can be obtained by using polynomial model, which was derived using optimal non-uniform delay coordinates. This technique is based on combination of non-uniform embedding, evolutionary optimization algorithms and polynomial model. For identification of embedding dimension False Nearest Neighbor method was used, which is the easy and fast way to find minimal embedding dimension. For identification of set of non-uniform time delays three different evolutionary algorithms were used. The found set was used as a time delay kernel for polynomial construction. Synchronization was carried for two different real world vibrations. The proposed model showed good synchronization results.

\section{Acknowledgements}

Financial support from Lithuania Science Council under Project No. MIP-078/2016 is acknowledged. 


\section{References}

[1] Yang S. S., Duan C. K. Generalized synchronization in chaos systems. Chaos, Solitons and Fractals, Vol. 9, Issue 10, 1998, p. 1703-1707.

[2] Maes K., Reynders E., Rezayat A., De Roeck G., Lombaert G. Offline synchronization of data acquisition systems using system identification. Journal of Sound and Vibration. Vol. 381, 2016, p. 264-272.

[3] Wu C. W., Chua L. O. A unified framework for synchronization and control of dynamical systems. International Journal of Bifurcation and Chaos, Vol. 4, Issue 4, 1994, p. 979-998.

[4] Lehnertz K., Bialonski S., Horstmann M. T., Krug D., Rothkegel A., Staniek M., Wagner T. Synchronization phenomena in human epileptic brain networks. Journal of Neural Science Methods, Vol. 183, 2009, p. 42-48.

[5] Jalili M. Spake phase synchronization in delayed - coupled neural networks: uniform vs. non-uniform transmission delay. Chaos, Vol. 23, Issue 1, 2013.

[6] Radhakrishnan S., Duvvuru A., Sultornsanee S. Phase synchronization based minimum spanning tree for analysis of financial time series with nonlinear correlations. Physica A, Vol. 444, 2016, p. 259-270.

[7] Schafer C., Rosenblum M. G., Kurths J., Abel H. H. Heartbeat synchronized with ventilation. Nature, Vol. 392, 1998, p. 239-240.

[8] Arnhold J., Grassberger P., Lehnertz K., Elger C. E. A robust method for detecting interdependences: application to intracranially recorded EEG. Physica D: Nonlinear Phenomena, Vol. 134, Issue 4, 1999, p. 419-430.

[9] DeShazer D. J., Breban R., Ott E., Roy R. Detecting phase synchronization in chaotic laser array. Physical Review Letters, Vol. 87, Issue 4, 2001, p. 044101.

[10] Pecora L. M., Carroll T. L. Synchronization in chaotic systems. Physical Review Letters, Vol. 84, Issue 8, 1990, p. 821-824.

[11] Faes L., Porta A., Nollo G. Mutual nonlinear prediction as a tool to evaluate coupling strength and directionality in bivariate time series: comparison among different strategies based on $\mathrm{k}$ nearest neighbors. Physical Review E, Vol. 78, 2008, p. 026201.

[12] Nichkawde C. Sparse model from optimal nonuniform embedding of time series. Physical Review E, Vol. 89, Issue 4, 2014, p. 042911.

[13] Sun Yeong-Jeu A novel chaos synchronization of uncertain mechanical systems with parameter mismatchings, external excitations, and chaotic vibrations. Communication in Nonlinear Science and Numerical Simulation, Vol. 17, 2012, p. 496-504.

[14] Awrejcewicz J., Krysko A. V., Yakovleva T. V., Zelenchuk D. S., Krysko V. A. Chaotic synchronization of vibrations of a coupled mechanical system consisting of a plate and beams. Latin American Journal of Solids and Structures, Vol. 10, 2013, p. 161-172.

[15] Fradkov A., Tomchina O., Galitskaya V., Gorlatov D. Multiple controlled synchronization for 3-rotor vibration unit with varying Payload. IFAC Proceeding Volumes, Vol. 46, Issue 12, 2013, p. 5-10.

[16] Ragulskis M., Lukoseviciute K. Non-uniform attractor embedding for time series forecasting by fuzzy inference systems. Neurocomputing, Vol. 72, 2009, p. 2618-2626.

[17] Lukoseviciute K., Ragulskis M. Evolutionary algorithms for the selection of time lags for time series forecasting by fuzzy inference systems. Neurocomputing, Vol. 73, 2010, p. 2077-2088.

[18] Theiler J. Efficient algorithm for estimating the correlation dimension from a set of discrete points. Physical Review A, Vol. 36, Issue 9, 1987, p. 4456-4462.

[19] Maus A., Sprott J. C. Neural network method for determining embedding dimension of a time series. Communication in Nonlinear Science and Numerical Simulation, Vol. 16, 2011, p. 3294-3302.

[20] Rhodes C., Morari M. False-nearest-neighbors algorithm and noise-corrupted time series. Physical Review E, Vol. 55, Issue 5, 1997, p. 6162-6170.

[21] Cao L. Practical method for determining the minimum embedding dimension of a scalar time series. Physica D: Nonlinear Phenomena, 1997, p. 43-50.

[22] Su Li-yun Prediction of multivariate chaotic time series with local polynomial fitting. Computers and Mathematics with Applications, Vol. 59, 2010, p. 734-744.

[23] Varadan V., Leung H. Reconstruction of polynomial systems from noisy time-series measurements using genetic programming. IEEE Transactions on Industrial Electronics, Vol. 48, Issue 4, 2001, p. $742-748$. 
[24] Neri Igor, et al. A real vibration database for kinetic energy harvesting application. Journal of Intelligent Material Systems and Structures, Vol. 23, 2012, p. 2095-2101.

[25] Bechhoefer E. High Speed Gear Dataset. Acknowledgement is made for the measurements used in this work provided through data-acoustics.com Database.

[26] Holland J. H. Adaptation in Neural and Artificial Systems: An Introductory Analysis with Application to Biology, Control and Artificial Intelligence. The MIT Press, 1992.

[27] Man K. F., Tang K. S., Kwong S. Genetic algorithms: concepts and applications. IEEE Transactions on Industrial Electronics, Vol. 43, Issue 5, 1996, p. 519-534.

[28] Karaboga D., Basturk B. On the performance of artificial bee colony (abc) algorithm. Applied Soft Computing, Vol. 8, 2008, p. 687-697.

[29] Karaboga D., Gorkemli B., Ozturk C., Karaboga N. A Comprehensive survey: artificial bee colony (ABC) algorithm and applications. Artificial Intelligence Review, Vol. 42, Issue 1, 2014, p. 21-57.

[30] Rajabioun R. Cuckoo optimization algorithm. Applied Soft Computing, Vol. 11, 2011, p. 5508-5518.

[31] Rakhshani H., Dehghanian E., Rahati A. Hierarchy Cuckoo search algorithm for parameter estimation in biological systems. Chemometrics and Intelligent Laboratory Systems, Vol. 159, 2016, p. 97-107.

[32] Draper N., Smith H. Applied regression analysis. Biometrical Journal, John Wiley and Sons, Vol. 11, Issue 6, 1969.

[33] Candan C. A unified framework for derivation and implementation of Savitzky-Golay filters. Signal Processing, Vol. 104, 2014, p. 203-211.

\section{Appendix}

\section{A1. Model for Case 1 system}

The 4-th order polynomial for the vibration of aquarium with air pump:

$$
\begin{aligned}
x_{i+1}^{(4)} & =0.1662-9.5534 X_{1 i}-6.8198 X_{2 i}+7.9247 X_{3 i}-6.7640 X_{4 i}-6.5820 X_{5 i} \\
& -5.3376 X_{6 i}+178.1441 X_{1 i} X_{2 i}-49.4790 X_{1 i} X_{3 i}+101.5937 X_{1 i} X_{4 i}+96.9213 X_{1 i} X_{5 i} \\
& +252.8560 X_{1 i} X_{6 i}-372.0087 X_{2 i} X_{3 i}+610.4346 X_{2 i} X_{4 i}-203.2786 X_{2 i} X_{5 i} \\
& +230.4250 X_{2 i} X_{6 i}-684.9584 X_{3 i} X_{4 i}+537.3870 X_{3 i} X_{5 i}-503.9905 X_{3 i} X_{6 i} \\
& +133.7229 X_{4 i} X_{5 i}+156.6968 X_{4 i} X_{6 i}+397.3481 X_{5 i} X_{6 i}+153.4929 X_{1 i}^{2} \\
& +83.6710 X_{2 i}^{2}+173.0346 X_{3 i}^{2}+138.5126 X_{4 i}^{2}-200.0438 X_{5 i}^{2}-48.2852 X_{6 i}^{2} \\
& +0.9830 x_{i+1}^{(2)}+0.1674 X_{1 i} x_{i+1}^{(2)}+0.0416 X_{2 i} x_{i+1}^{(2)}+0.3283 X_{3 i} x_{i+1}^{(2)} \\
& -0.3190 X_{4 i} x_{i+1}^{(2)}+0.5565 X_{5 i} x_{i+1}^{(2)}+0.3720 X_{6 i} x_{i+1}^{(2)}+0.4029\left(x_{i+1}^{(2)}\right)^{2},
\end{aligned}
$$

where $\left(X_{1 i}, X_{2 i}, X_{3 i}, X_{4 i}, X_{5 i}, X_{6 i}\right)$ are time delay vectors, $x_{i+1}^{(2)}$ is second order polynomial found by Eq. (10) and $x_{i+1}^{(4)}$ is the fourth order polynomial found by Eq. (11) and $i=N+1, N+2, \ldots, K$.

\section{A2. Model for Case 2 system}

The 6-th order polynomial for the radial vibration of wind turbine pinion gear:

$$
\begin{aligned}
x_{i+1}^{(6)} & =0.4144-0.3828 X_{1 i}-0.6073 X_{2 i}-0.4430 X_{3 i}+0.4618 X_{4 i}+0.1722 X_{1 i} X_{2 i} \\
& +0.1178 X_{1 i} X_{3 i}-0.1320 X_{1 i} X_{4 i}+0.2657 X_{2 i} X_{3 i}-0.0592 X_{2 i} X_{4 i} \\
& -0.2137 X_{3 i} X_{4 i}+0.0935 X_{1 i}^{2}+0.1940 X_{2 i}^{2}+0.0812 X_{3 i}^{2}-0.0962 X_{4 i}^{2} \\
& +1.1978 x_{i+1}^{(2)}-0.0493 X_{1 i} x_{i+1}^{(2)}+0.0561 X_{2 i} x_{i+1}^{(2)}-0.0294 X_{3 i} x_{i+1}^{(2)} \\
& +0.0504 X_{4 i} x_{i+1}^{(2)}-0.1259\left(x_{i+1}^{(2)}\right)^{2}+0.9526 x_{i+1}^{(4)}+0.0036 X_{1 i} x_{i+1}^{(4)} \\
& -0.0005 X_{2 i} x_{i+1}^{(4)}+0.0127 X_{3 i} x_{i+1}^{(4)}-0.0249 X_{4 i} x_{i+1}^{(4)}+0.0554\left(x_{i+1}^{(4)}\right)^{2}
\end{aligned}
$$

where $\left(X_{1 i}, X_{2 i}, X_{3 i}, X_{4 i}\right)$ are time delay vectors, $x_{i+1}^{(2)}$ is second order polynomial found 
by Eq. (10), $x_{i+1}^{(4)}$ and $x_{i+1}^{(6)}$ are fourth and sixth order polynomials found by Eq. (11) and Eq. (12) and $i=N+1, N+2, \ldots, K$.

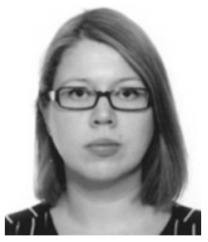

Migle Drulyte received M.S. degree in mathematics from Kaunas University of Technology, Kaunas, Lithuania, in 2013. She is currently a Ph.D. student at the Department of Mathematical Modelling, Kaunas University of Technology. Her research interest: optimization algorithms; evolutionary computations; numerical analysis.

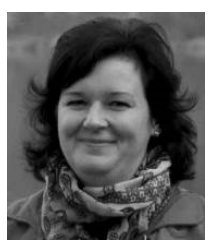

Kristina Lukoseviciute received the Ph.D. degree in Informatics from Kaunas University of Technology, Lithuania in 2012. Since 2014 she is an Associate Professor at the Department of Mathematical Modelling, Kaunas University of Technology. Her current research interests are evolutionary computations; time series analysis; time of flight estimation.

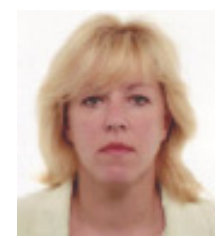

Jurate Ragulskiene is a docent at the Department of Mathematical Modelling, Kaunas University of Technology, Kaunas, Lithuania. Her areas of interest cover nonlinear and evolutionary dynamics, numerical analysis and computational technology. 